\title{
GPPS-CH-2020-134
}

\section{EXTRACTION AND VISUALIZATION OF VORTICAL FLOW FEATURES IN A HIGHLY LOADED COMPRESSOR CASCADE}

\author{
Yangwei Liu \\ 1. National Key Laboratory of \\ Science and Technology on Aero- \\ Engine Aero-Thermodynamics, \\ School of Energy and Power \\ Engineering, Beihang University \\ 2. Collaborative Innovation \\ Center of Advanced Aero-Engine, \\ Beihang University \\ Email: liuyangwei@126.com \\ Beijing 100191, China
}

\author{
Zhaoqing Cheng \\ National Key Laboratory of \\ Science and Technology on Aero- \\ Engine Aero-Thermodynamics, \\ School of Energy and Power \\ Engineering, Beihang University \\ Email: czq@buaa.edu.cn \\ Beijing 100191, China
}

\author{
Yumeng Tang* \\ National Key Laboratory of \\ Science and Technology on Aero- \\ Engine Aero-Thermodynamics, \\ School of Energy and Power \\ Engineering, Beihang University \\ Email: tang_yumeng@126.com \\ Beijing 100191, China \\ ${ }^{*}$ Corresponding author
}

\begin{abstract}
Vortex identification and visualization play a significant role in studying the turbulence or flow mechanisms, turbulence models, and flow control approaches. While rarely works have been done on evaluating different vortex identifications in complex practical flow field. The current research extracts the vortical flow features in a highly loaded compressor cascade at $\mathrm{Ma}=0.59$ using different vortex identification methods, including the Helmholtz decomposition series and the eigen decomposition series. The analytic solutions for different vortex identifications are derived, and the inter-relationship between the Helmholtz decomposition series and the eigen decomposition series is built theoretically through $L T_{c r i}$ associates with the projection plane constructed for it. Results show that vortices in the practical flow field tend to overlap with each other and have irregular edges. All the methods compared are capable of extracting vortex structures in their theoretical threshold bounds, while their effect of confining vortex through non-zero threshold are not necessarily the same. Capacities of different vortex identifications are elaborately compared and discussed.
\end{abstract}

\section{INTRODUCTION}

Vortex acts as a manner that the flow accumulates energy in a narrow space. Despite there is none mathematical definition of vortex that has been accepted yet, vortex is widely investigated in various flow phenomena. The compressor blade passage is a typical curved diffusion passage with small internal space, thus, large adverse pressure gradient is suffered. Complex vortex structures exist in the blade passage of compressor due to the effect of viscosity, such as horseshoe vortex (HV), passage vortex (PV), corner vortex (CV), concentrated shedding vortex (CSV), et al. The formation, development, breakdown, dissipation process of vortices, interaction among different vortices, and interaction of vortices with main flow makes flow extraordinarily complex. The secondary flow loss caused thereby, significantly restricts the improvement of compressor performance (Liu, et al. 2017).

During the past several decade, a great deal of works have done on the recognition of vortices within the compressor cascade passage. Kang (1993) investigated the vortex structures of the three-dimensional (3D) corner separation using topology technique, and illustrated the complex vortex structures that compose the $3 \mathrm{D}$ corner separation of the compressor cascade. Among all the vortices, the PV is the most prominent one, and it owns the largest scale. Herzig (1954) firstly detached the PV structure in the compressor cascade passage through visible plume tracer technique in experiment. Klein (1966) proposed the HV structure near the blade leading-edge in 1966, and named it as HV since its two branch looks like horseshoe. The existence of blade leading-edge splits the incoming flow, and formulates partially separation, thus, creating the HV. The formation of HV is closely related to the incoming flow velocity, boundary layer thickness, and blade leading-edge shape. However, the small scale of the HV that detached in the practical case, makes it hard to be measured exactly by experiments, and confused to be separated from complex vortices of 3D corner separation flow in numerical analyses. The development of blade suction side branch and pressure side branch of HV is still 
controversial. Gbadebo et al. (2005) held the view that the suction side branch of HV rolls into the 3D corner separation structures formed in the blade endwall conjunction, then develops downstream with the complex vortices that composes the 3D corner separation until the take-off from the blade trailing-edge. For the pressure side branch of HV, it is thought to be immerged into PV from the majority, and PV is thought to be sharing the same sense of rotating with pressure side branch of HV. CV is observed by Gregory and Grave in 1983 (Gregory and Grave, 1983) in experiments by the underturning of flow in the exit of blade passage near the endwall. It is relatively tiny in the 3D corner separation structures, and it forms in the conjunct corner of the blade suction surface and the endwall, and develops downstream closely to the conjunct corner. Some perspective assumed that it had been induced by PV, since they share opposite sense of rotating. The shedding vortices in the blade trailing-edge comes from the interaction of two individual boundary layer from blade suction surface and pressure surface under the adverse pressure gradient effect. It is divided into free shedding vortex and concentrated shedding vortex according to its pattern. The free shedding vortex mainly forms in the blade middle region by wake, and the concentrated shedding vortex is caused by the rolling up of migrated boundary layer flow along the blade suction corner from the endwall region under the adverse pressure gradient.

Earlier recognition of 3D flow structures in the compressor blade passage are mainly based on the experimental observation intuitively, such as smoke-wire visualization or oil visualization. Topology diagnosis is commonly applied with oil visualization, and it is also popular in the analysis of wall limiting strace of CFD results (Gbadebo et al., 2005). However, the topology deduced by wall limiting strace misses out the flow information in the perpendicular dimension, thus it is inaccurate to reflect the secondary flow structures in the blade passage. Considering the secondary flow and its loss relate directly to the generation and evolution of vortices, it is of great meaning to gain deeper insight into the complex vortex structures within the compressor passage (Tang, et al. 2018). The choice of proper vortex identification methods that suitable for the analysis of compressor flow is of great importance. An accurate method assists in establishing vivid concept of complex separation structures within the compressor passage, thereby contributes to the exploration of 3D corner separation mechanism and promotes the aerodynamic improvement corresponding. Epps (2017) conducted a review of commonly used vortex identification methods, and divided them into four types. Among them, the region type method distinguishes the vortex through comparing a scalar function to a certain threshold. Most of region type methods are derived from velocity gradient tensor, and work pointwise. These include the secondary invariant of velocity gradient tensor (known as Q-criterion (Hunt et al., 1988)), the discriminant of velocity gradient tensor (known as $\Delta$ criterion (Chong et al., 1990)), and the eigen decomposition series (e.g. $\lambda_{c i}$ swirling-strength criterion (Berdahl et al., 1993), $\lambda_{c r} / \lambda_{c i}$ enhanced swirling-strength criterion (Chakraborty et al., 2005)). Although a great deal of vortex identification methods have been proposed, little attention has been paid to performing a comprehensive comparison of these methods, especially for the 3D corner separation flow in compressors.

The current research derives and compares different vortex identification methods for extracting vortices in a highly loaded compressor cascade passage. The delayed detached eddy simulation (DDES) is conducted. The density weighted sampled flow fields are analysed. Part 2 describes the investigated blade geometry, computational settings and provides the validation of sampled flow field to the experimental results. Part 3 presents the analytic solutions for the commonly used vortex identification methods, especially for the Q-criterion, two variation forms of Q-criterion, and the eigen decomposition based methods. The inter-relationship of these identification methods are explored mathematically. Part 4 compares the application of different vortex identifications to the typical stream surfaces of a highly loaded compressor cascade, and discusses the capacity of different identifications with varied thresholds. Part 5 remarks the major finds.

\section{COMPUTATIONAL SETUP AND VALIDATION}

DDES is conducted on the highly loaded compressor cascade that was experimentally tested by us previously. The experiments were conducted over a wide range of incidence angles at $\mathrm{Ma}=0.59$. More details about the cascade experiments could refer to Tang Y., Liu Y., et al. (2017 \& 2020). Only the datum cascade under the incoming flow angle of $0^{\circ}$ is investigated in this study based on experimental calibrated DDES results.

\subsection{Geometrical Model}

The cascade profile investigated is a cut-off of a state-of-art compressor stator. A linear geometry model is constructed the same as experiments. Geometric parameters of the cascade are shown in Table 1.

Table 1 Geometric Parameters of Investigated Cascade

\begin{tabular}{llcccc}
\hline \hline Parameter & Value & Units & Parameter & Value & Units \\
\hline Chord $/ c$ & 0.055 & {$[\mathrm{~m}]$} & Stagger Angle & 21.7 & {$\left[{ }^{\circ}\right]$} \\
Solidity $(c / t)$ & 1.52 & {$[-]$} & Camber Angle & 52.94 & {$\left[{ }^{\circ}\right]$} \\
Aspect Ratio $(h / c)$ & 1.82 & {$[-]$} & Outlet Angle & 94.85 & {$\left[{ }^{\circ}\right]$} \\
Inlet Angle & 41.91 & {$\left[{ }^{\circ}\right]$} & Design Ma & 0.59 & {$[-]$} \\
\hline
\end{tabular}


Figure 1 provides the sketch of computational domain. Only one blade passage is modelled in the numerical simulation with the periodic boundary condition used in two side wall of the computational domain. The cascade is tested without tip clearance, so the blade middle plane of the computational domain is set as symmetric plane. The incoming flow measurements are conducted $1 c$ ahead of the blade leading-edge, and the inlet position of the computational domain keeps the same with experimental measurement. The total pressure and flow angle distribution measured in experiments are set as the inlet flow boundary conditions. The outlet of the computational domain is set $3 c$ behind of the blade trailing-edge to make the flow fully mixed. All the wall is set as no-slip wall. And the Mach number simulated remains the same as experiments, which values 0.59 .

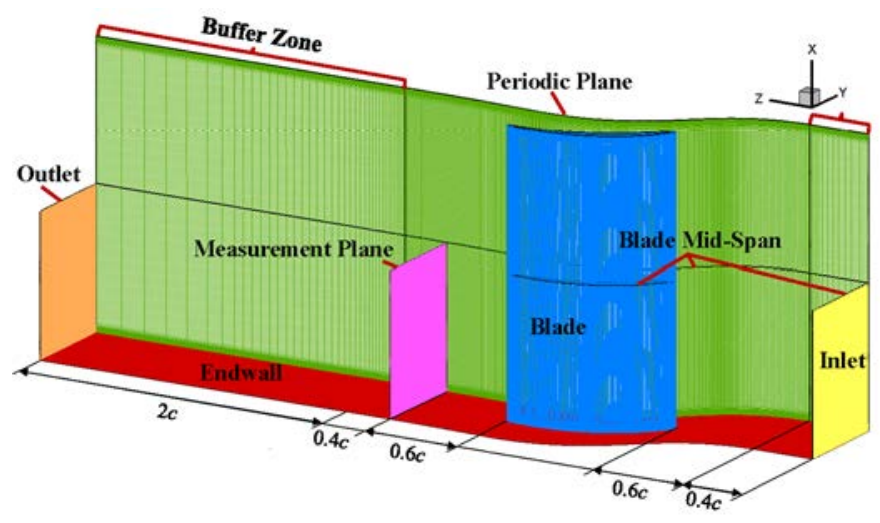

Figure 1 Computational Domain

The H-O4H-H multi-block grid strategy has been utilized with seven grid blocks. The wall $y^{+}$is set as $y^{+}<1$ to guarantee the flow within the boundary layer fully resolved. The maximum grid scale in the spanwise, and streamwise are $\Delta x^{+}<180$, and $\Delta z^{+}<200$, and it satisfies the proposal for grid requirement of DDES by Tucker (Tucker, 2013). The total grid points is approximate 9.17 million for half span height. The region that covers $60 \% c$ ahead of the blade leading-edge and $1 c$ behind the blade trailing-edge is regarded as core resolved region. Thus, buffer zone is utilized to make the fine grid concentrated on the core resolved region and to avoid reflection.

\subsection{Computational Procedure}

The Spalart-Allmaras (SA) model based DDES method is utilized in this study. And the FLUENT software is used as solver. The incoming flow is homogeneous fully turbulent in the experiment, so none perturbation is given for the inlet boundary condition. The physical time step is set as

$$
\Delta t=\frac{1}{1000} \frac{c}{U_{\text {ref }}} \approx 2.1418 \times 10^{-7} \mathrm{~s}
$$

Where $U_{\text {ref }}$ stands for the bulk flow velocity of the incoming flow.

128 cores are used for parallel computation. Totally 12 thousand time steps are sampled for the time-averaged flow field after it achieves statistical stationary.

\subsection{Validation of the Sampled Flow-Field}

Considering compressibility of the flow, the density weighted average is utilized in processing the averaged flow field. Figure 2 compares the total pressure loss contours in the measurement plane $60 \% \mathrm{c}$ downstream the blade trailingedge.

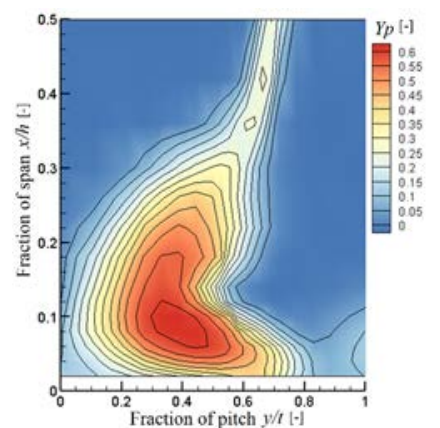

(a) Experimental measurement

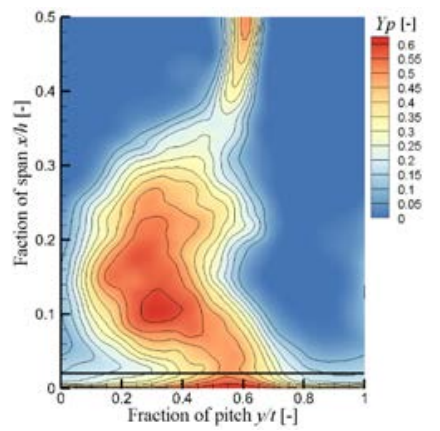

(b) Sampled Flow Field

Figure 2 Comparison of Total Pressure Loss Contours in the Measurement Plane 
Figure 2 shows that the DDES sampled flow field provides similar separation shape to the experimental result. Although related to the experimental result, the high loss core of the sampled result is slightly lift-off from endwall, and the loss magnitude in the wake centre is larger, generally, the DDES sampled result provides considerable extend and tendency of separated regions as seen in the experiment. Thus the simulation is reliable, and it could provide more details about the flow field that is hard to measure experimentally.

\section{ANALYTIC SOLUTION OF VORTEX IDENTIFICATION METHODS}

\subsection{Q-criterion and its variation form}

The well-known Q-criterion is derived based on the incompressible flow, and it is widely used in many practical flow cases (Liu, et al. 2019b). While, the flow in the current research is compressible. Thus, Q-criterion and its two variation forms for compressible flow are compared. $\Delta$ criterion and the eigen decomposition series methods are free from the influence of compressibility. $\Delta$ criterion acts as a division to segment the swirling and non-swirling zones. It determines whether the velocity gradient tensor has complex eigenvalues. The $\lambda_{c i}$ swirling-strength criterion utilizes the imaginary part of the eigenvalue to visualize the vortex. Recently, a local trace criterion $\left(L T_{c r i}\right)$ is proposed by Liu Y. and Tang Y. (2019). It is derived as an eigen decomposition series method, and it successfully connects to the mission of Qcriterion while overcomes the restriction of compressibility. Here, the analytical expression for Q-criterion and its two variation forms for compressible flow, $\Delta$ criterion and the eigen decomposition series methods, and $L T_{c r i}$ are given.

The Q-criterion named by the secondary invariant of velocity gradient tensor. And it takes

$$
Q=I I_{\nabla \boldsymbol{u}}=\frac{1}{2}\left[(\operatorname{tr}(\nabla \boldsymbol{u}))^{2}-\operatorname{tr}\left((\nabla \boldsymbol{u})^{2}\right)\right]
$$

Where $\operatorname{tr}(\nabla \boldsymbol{u})$ stands for the trace of velocity gradient tensor.

For the incompressible flow, the first invariant of velocity gradient tensor, $P=I_{\nabla \boldsymbol{u}}=-\operatorname{tr}(\nabla \boldsymbol{u})=0$. Thus, it achieves $Q=\left(\|\boldsymbol{\Omega}\|^{2}-\|\boldsymbol{S}\|^{2}\right) / 2$. However, for the compressible flow, the non-zero $P$ makes $Q \neq\left(\|\boldsymbol{\Omega}\|^{2}-\|\boldsymbol{S}\|^{2}\right) / 2$. A variation from of Q-criterion, $Q_{D}$, is proposed by Kolár (2009) to account for the compressibility effect. When the dilatation does not play a role, the criterion only depends on the deviatoric part, thus $Q_{D}$ reads as

$$
Q_{D}=\left(\|\boldsymbol{\Omega}\|^{2}-\left\|\boldsymbol{S}_{\boldsymbol{D}}\right\|^{2}\right) / 2
$$

$\boldsymbol{\Omega}$ is antisymmetric component of velocity gradient tensor, $\nabla \boldsymbol{u}$. And

$$
\boldsymbol{\Omega}=\frac{1}{2}\left(\nabla \boldsymbol{u}-\nabla \boldsymbol{u}^{\boldsymbol{T}}\right)
$$

$\boldsymbol{S}_{\boldsymbol{D}}$ stands for the deviatoric part of the strain rate tensor, and it takes the following form.

$$
\begin{aligned}
& \boldsymbol{S}_{\boldsymbol{D}}=\boldsymbol{S}-\frac{1}{3} \operatorname{tr}(\boldsymbol{S}) \cdot \boldsymbol{I} \\
& \boldsymbol{S}=\frac{1}{2}\left(\nabla \boldsymbol{u}+\nabla \boldsymbol{u}^{\boldsymbol{T}}\right)
\end{aligned}
$$

Kolár̆ and Š́stek (2015) noticed that when investigate the velocity gradient in the frame of strain-rate principal axes, it could be divided as a half vorticity vector, $\boldsymbol{\omega}=(\nabla \times \boldsymbol{u}) / 2$ and a principal strain-rate difference vector $\boldsymbol{\sigma}$.

$$
\nabla \boldsymbol{u}=\left[\begin{array}{ccc}
\sigma_{1} & -\omega_{3} & \omega_{2} \\
\omega_{3} & \sigma_{2} & -\omega_{1} \\
-\omega_{2} & \omega_{1} & \sigma_{3}
\end{array}\right]
$$

$\boldsymbol{\sigma}=\left(\sigma_{2}-\sigma_{3}, \sigma_{3}-\sigma_{1}, \sigma_{1}-\sigma_{2}\right) / 2$ is free of the effect of expansion and compression. Thus, another variant of Qcriterion, $Q_{M}$, is defined as

$$
Q_{M}=|\omega|^{2}-|\sigma|^{2}
$$

\subsection{Analytic solution for eigen decomposition series methods}

For the eigen decomposition series methods, let $A_{i j}=\partial u_{i} / \partial x_{j}(i, j=1,2,3)$ be the velocity gradient tensor for the 3D flow field. Namely, $\boldsymbol{A}=\nabla \boldsymbol{u}$, and $A v=\lambda v$ has three eigenvalues and three corresponding eigenvectors. Deriving from the critical point theory, the local perturbation at the point has a tendency for swirling (also for rotation) when a couple of conjugate complex eigenvalues exists for $\boldsymbol{A}$. In this case, the velocity gradient tensor could be expressed as

$$
\boldsymbol{A}=\nabla \boldsymbol{u}=\left[\begin{array}{lll}
\boldsymbol{v}_{r} & \boldsymbol{v}_{c r} & \boldsymbol{v}_{c i}
\end{array}\right]\left[\begin{array}{ccc}
\lambda_{r} & & \\
& \lambda_{c r} & \lambda_{c i} \\
& -\lambda_{c i} & \lambda_{c r}
\end{array}\right]\left[\begin{array}{lll}
\boldsymbol{v}_{r} & \boldsymbol{v}_{c r} & \boldsymbol{v}_{c i}
\end{array}\right]^{-\mathbf{1}}
$$


$\lambda_{r}$ is the real eigenvalue for $\nabla \boldsymbol{u}, \lambda_{c r}$ and $\lambda_{c i}$ is the real part and the imagine part of the conjugate complex eigenvalues, respectively.

Traditionally, eigenvalues are resolved by cumbersome matrix manipulations, thus, it is hard to be parallelly processed with massive grid points. Here, the analytic solutions for the real eigenvalue as well as the real and imagine components of conjugate complex eigenvalues are derived through Shengjin's formulas (Fan S.J., 1989).

Let $P, Q, R$ be the three invariants of velocity gradient tensor, $\nabla \boldsymbol{u}$. Eq. (2) provides the expression for the second invariant, $Q$. The first invariant, $P$, reflects the divergence of the flow, and it takes $P=-\operatorname{tr}(\nabla \boldsymbol{u})$. The third invariant, $R$, is opposite to the determinant of velocity gradient tensor, $R=-\operatorname{det}(\nabla \boldsymbol{u})$.

$\Delta$ acts as a judgement to discriminate whether the velocity gradient tensor has complex eigenvalues. The positive $\Delta$ implies the existence of complex eigenvalues, and a swirling tendency for the local flow point. It could be expressed as a function of $P, Q, R$.

$$
\Delta=\left[\left(\frac{Q}{3}-\frac{P^{2}}{9}\right)^{3}+\left(\frac{P Q}{6}-\frac{R}{2}-\frac{P^{3}}{27}\right)^{2}\right]
$$

Introducing two auxiliary variables, $Y_{1}$ and $Y_{2}$, when $\Delta>0$.

$$
\begin{aligned}
& Y_{1}=P^{3}-\frac{9}{2} P Q+\frac{27}{2} R+27 \sqrt{\Delta} \\
& Y_{2}=P^{3}-\frac{9}{2} P Q+\frac{27}{2} R-27 \sqrt{\Delta}
\end{aligned}
$$

The analytical formulation for $\lambda_{r}, \lambda_{c r}$, and $\lambda_{c i}$, when $\Delta>0$, are expressed as following.

$$
\begin{gathered}
\lambda_{r}=-\frac{1}{3} P-\frac{1}{3}\left(\sqrt[3]{Y_{1}}+\sqrt[3]{Y_{2}}\right) \\
\lambda_{c r}=-\frac{1}{3} P+\frac{1}{6}\left(\sqrt[3]{Y_{1}}+\sqrt[3]{Y_{2}}\right) \\
\lambda_{c i}=\frac{\sqrt{3}}{6}\left(\sqrt[3]{Y_{1}}-\sqrt[3]{Y_{2}}\right)
\end{gathered}
$$

With the analytical solutions for $\lambda_{r}, \lambda_{c r}$, and $\lambda_{c i}$ given, the eigen decomposition series vortex identification methods, like $\lambda_{c i}$ swirling-strength criterion, $\lambda_{c r} / \lambda_{c i}$ enhanced swirling-strength criterion, could be easily resolved pointwise, and the cumbersome matrix manipulations to resolve eigenvalues are avoided.

\subsection{Review of the $L T_{c r i}$}

The $L T_{c r i}$ (Liu Y. and Tang Y., 2019a) extracts the vortex through the sign determination of the trace of a new tensor, $\boldsymbol{\Psi} . \boldsymbol{\Psi}$ is constructed as half of the quadratic velocity gradient, $\nabla \boldsymbol{u}$. Thus, the $L T_{c r i}$ reads as

$$
L T_{c r i}=-\frac{1}{2} \lambda_{r}^{2}-\lambda_{c r}^{2}+\lambda_{c i}^{2}, \Delta>0
$$

The physical interpretation of $L T_{c r i}$ is unambiguous, it extracts the swirling dominated region both for the compressible and incompressible flow. Further, a projection plane with a divided elliptical region is constructed associated with $L T_{c r i}$. The projection plane is also named as positive-Delta space, for all the flow point that achieves $\Delta>$ 0 could find its unique position in the projection plane. The abscissa axis and the ordinate axis of the projection plane is defined as $\xi=\lambda_{r} /\left|\lambda_{c i}\right|, \eta=\lambda_{c r} /\left|\lambda_{c i}\right|$, respectively. Figure 3 illustrates the elliptical region that confined by $L T_{c r i}>0$ in the constructed positive-Delta space.

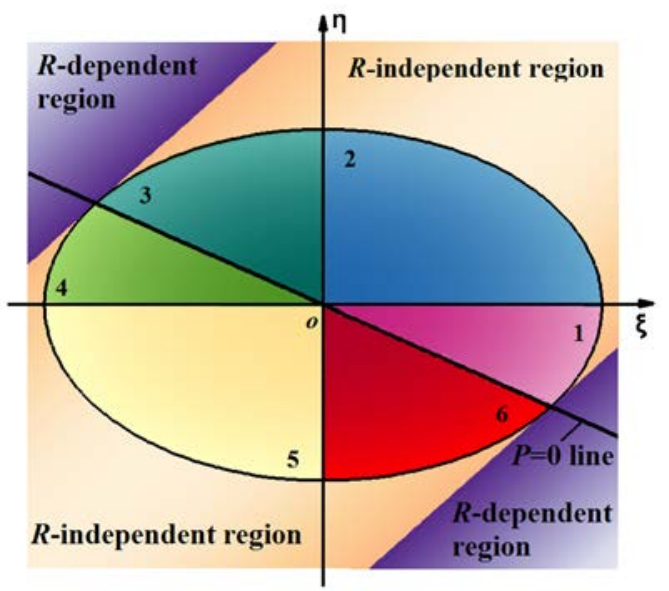

Figure 3 Elliptical Region Judged by $L T_{c r i}$ in the Projection Plane (Liu Y. and Tang Y., 2019) 
The $P=0$ iso-line divides the projection plane into two parts, regions above the $P=0$ iso-line $(P<0)$ implies the flow is in an expansion state, and regions below the $P=0$ iso-line $(P>0)$ denotes compression. For incompressible flow, whose $P=0$, all flow points are situated on the line. The region coloured by orange represents the $R$-independent region, and the purple represents the $R$-dependent regions. For more detailed introduction, it could refer to the Ref. by Liu Y. and Tang Y. (2019).

The elliptical region is divided into six parts, representing the combination of compressibility and different swirling patterns. Part 1, 2, and 3 represents expansion, and part 4, 5, and 6 represent compression. Additionally, as shown in Figure 4, flow points projected into different quadrants indicate different types of swirling trajectories.

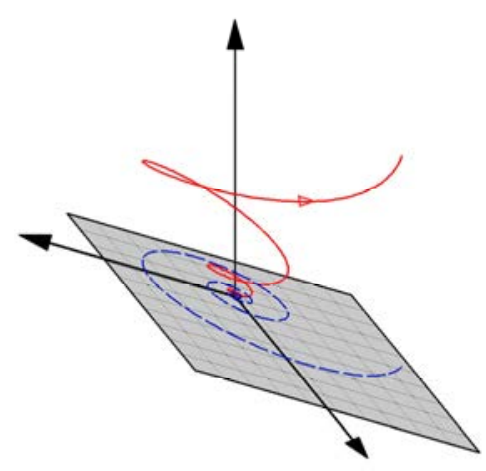

(a) Projection in Quadrant I

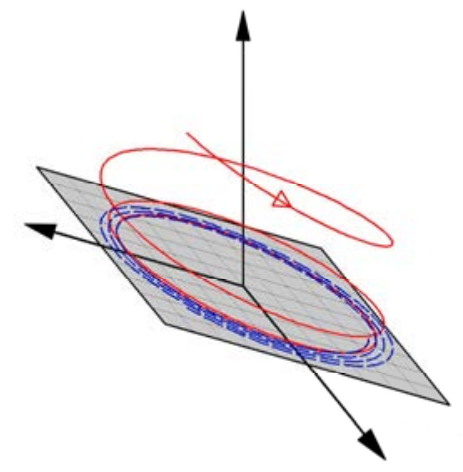

(c) Projection in Quadrant III

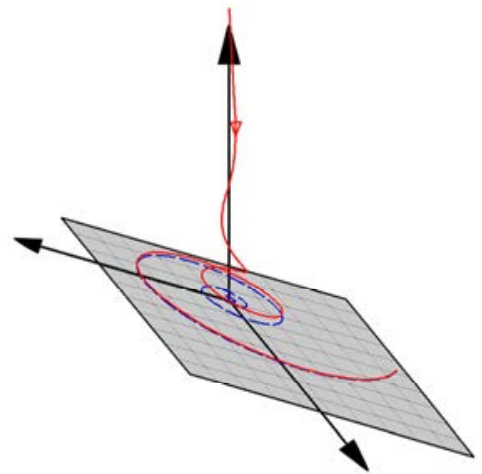

(b) Projection in Quadrant II

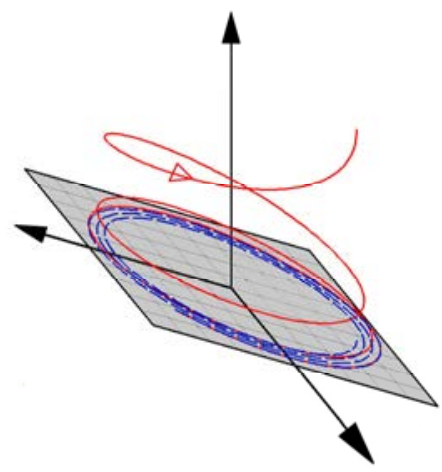

(d) Projection in Quadrant IV

Figure 4 Swirling Trajectories for Flow Points Projected into Different Quadrants

\subsection{Theoretical relationship of different identification methods}

Q-criterion is a typical method of Helmholtz decomposition of velocity gradient tensor, $\nabla \boldsymbol{u}$. Rarely work has done to explore the connection of vortex identification method based on Helmholtz decomposition and the eigen decomposition series vortex identification methods. The proposed $L T_{c r i}$ successfully provides a connection between Qcriterion and eigen decomposition series methods. From physical point of view, $L T_{c r i}>0$ also expressed as

$$
L T_{c r i}=\frac{1}{2}\left(\|\Omega\|^{2}-\|\boldsymbol{S}\|^{2}\right)=Q-\frac{P^{2}}{2}
$$

According to the constructed positive-Delta space shown in Figure 3, there exists

$$
\frac{1}{2}\left(\|\boldsymbol{\Omega}\|^{2}-\|\boldsymbol{S}\|^{2}\right)>0 \Leftrightarrow \frac{1}{2}\left(\frac{\lambda_{r}}{\lambda_{c i}}\right)^{2}+\left(\frac{\lambda_{c r}}{\lambda_{c i}}\right)^{2}<1
$$

And $L T_{c r i}>0$ equals to $\lambda_{c i}^{2}>\lambda_{r}^{2} / 2+\lambda_{c r}^{2}$.

$Q_{M}$ criterion is derived under the frame of strain-rate principal axes. The extension form of $Q_{M}$ is obtained as

$$
Q_{M}=\left(\omega_{1}^{2}+\omega_{2}^{2}+\omega_{3}^{2}\right)+\frac{1}{2}\left(\sigma_{1} \sigma_{2}+\sigma_{2} \sigma_{3}+\sigma_{1} \sigma_{3}\right)-\frac{1}{2}\left(\sigma_{1}^{2}+\sigma_{2}^{2}+\sigma_{3}^{2}\right)
$$

Substituting $\operatorname{tr}\left(\boldsymbol{S}^{2}\right)=\sigma_{1}^{2}+\sigma_{2}^{2}+\sigma_{3}^{2}$ and $\operatorname{tr}\left(\boldsymbol{\Omega}^{2}\right)=-2\left(\omega_{1}^{2}+\omega_{2}^{2}+\omega_{3}^{2}\right)$, it derives

$$
Q_{M}=L T_{c r i}+\frac{I I_{S}}{2}
$$

$I I_{S}$ is the second invariant of the strain rate tensor. 


\section{APPLICATION IN COMPRESSOR 3D CORNER SEPARATION FLOW}

To further investigate the similarity and difference of all these methods analysed above, comparisons are made in a practical flow case. The DDES dataset for a highly loaded compressor cascade under the Mach number of 0.59 are investigated. The density-weighted sampled flow field is analysed by different vortex identification methods.

The distribution of $\operatorname{tr}\left(\boldsymbol{S}^{2}\right)$ and $-\operatorname{tr}\left(\boldsymbol{\Omega}^{2}\right)$ in the S3 flow surface $20 \% c$ downstream the blade trailing-edge is provided in Figure 5 before the comparison of different comparison methods.

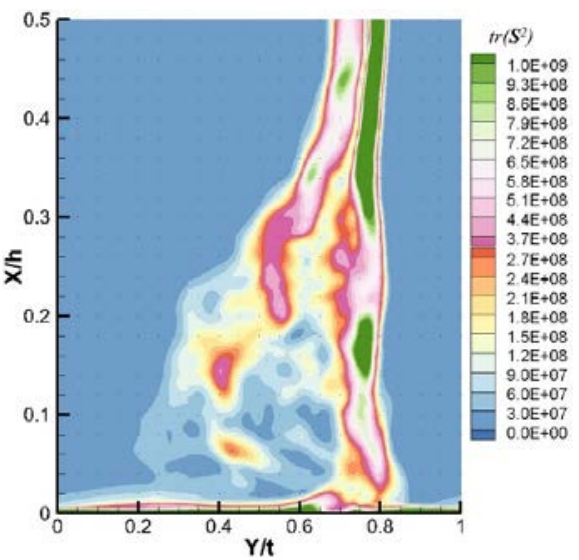

(a) $\operatorname{tr}\left(S^{2}\right)$

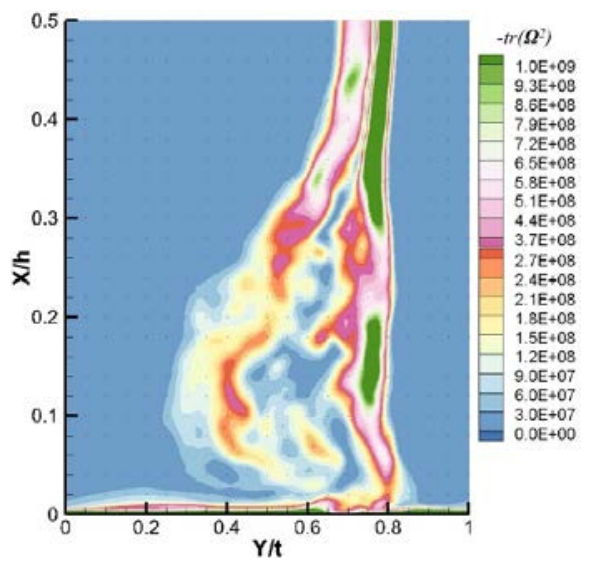

(b) $-\operatorname{tr}\left(\Omega^{2}\right)$

Figure 5 Distribution of $\operatorname{tr}\left(S^{2}\right)$ and $-\operatorname{tr}\left(\Omega^{2}\right)$ in S3 Stream Surface

The distribution of $\operatorname{tr}\left(\boldsymbol{S}^{2}\right)$ and $-\operatorname{tr}\left(\boldsymbol{\Omega}^{2}\right)$ agrees well in the wake region, and both of them obtain high values in the blade suction side wake. In the region adjacent to the endwall, the distribution of $\operatorname{tr}\left(\boldsymbol{S}^{2}\right)$ and $-\operatorname{tr}\left(\boldsymbol{\Omega}^{2}\right)$ extent also agrees well. The strain rate magnitude gets high values in the connected region of endwall corner and wake, covering approximate $20 \%$ to $30 \%$ span-height. Relatively, the vorticity magnitude in this region is lower than the strain rate magnitude. While, in the developing region of the corner separation towards the blade middle that within $20 \%$ spanheight, the vorticity magnitude is larger.

Corresponding, the vortex identification results by $Q$ criterion, $Q_{D}$ criterion, $Q_{M}$ criterion, $L T_{c r i}$ based elliptical region (ER) indication, $\lambda_{c i}$ swirling-strength criterion, and $\lambda_{c r} / \lambda_{c i}$ enhanced swirling-strength criterion in S3 stream surface $20 \% c$ downstream the blade trailing-edge are compared in Figure 6. Theoretically, the threshold for Q-criterion and two of its variation form is zero. Thus, values less than zero is blanked in Figure 6 (a), (b), (c), while the contour lines for the negative value is preserved as dash line. Legend for Figure 6 (c) is the projection plane shown in Figure 3 , only the elliptical region with six divisions that identified as vortex core region is shown. The $L T_{c r i}$ based ER indication is a good way to evaluate the disparity of different identifications.

Seen from Figure 6 (a), (b), the vortex bound that detached by zero threshold value of $Q_{D}$ criterion appears the same with $Q$ criterion results. While, differences exist in several regions when the same non-zero threshold is chosen. Figure 6 (c) shows that the judgement of $Q_{M}>0$ conspicuously shrinks the vortex bound in accordance to the results of $Q$ criterion and $Q_{D}$ criterion. Figure $6(\mathrm{~d})$ reflects that the vortex bound extracted by $L T_{c r i}>0$ is similar to the results of $Q$ criterion, while the $L T_{c r i}$ method indicates the small vortex region with different swirling patterns.

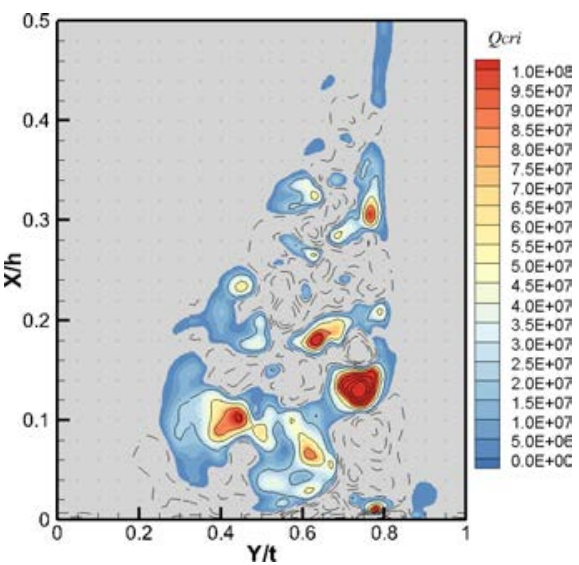

(a) $Q$ criterion

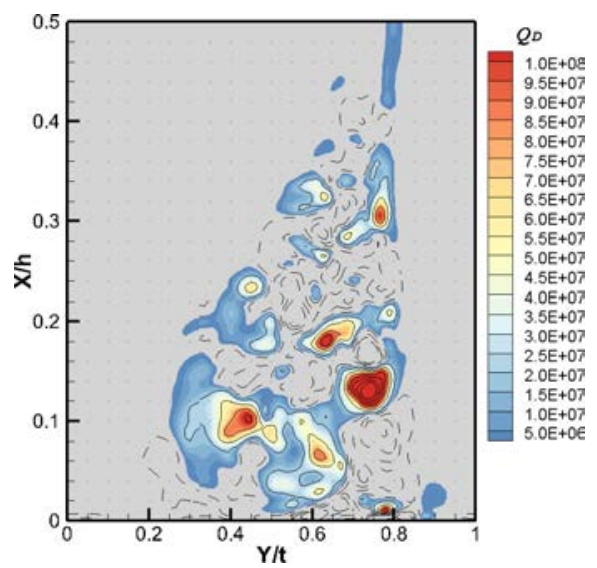

(b) $Q_{D}$ criterion 

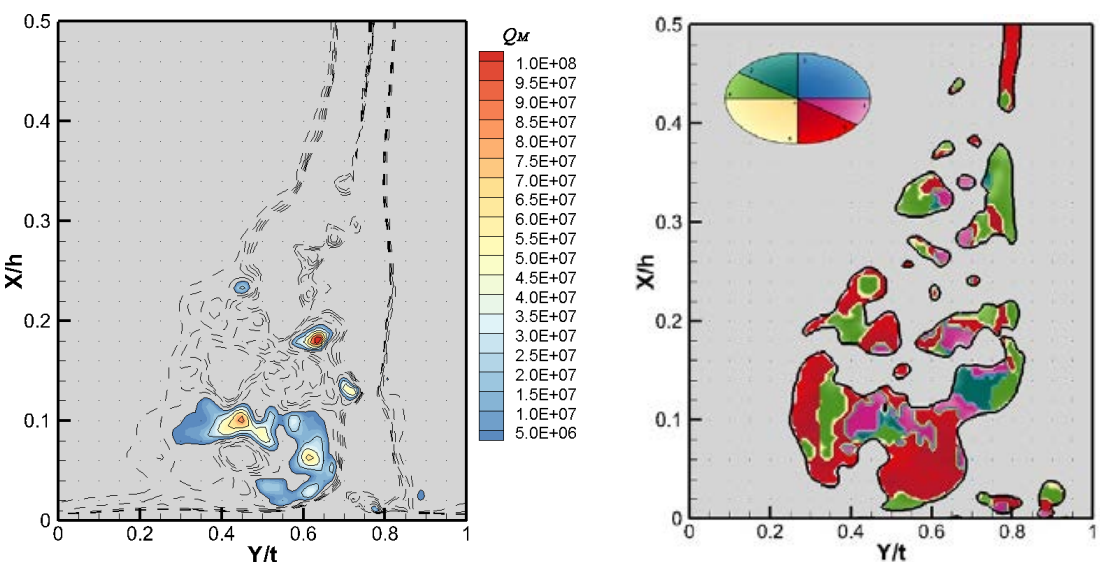

LTER

(c) $Q_{M}$ criterion

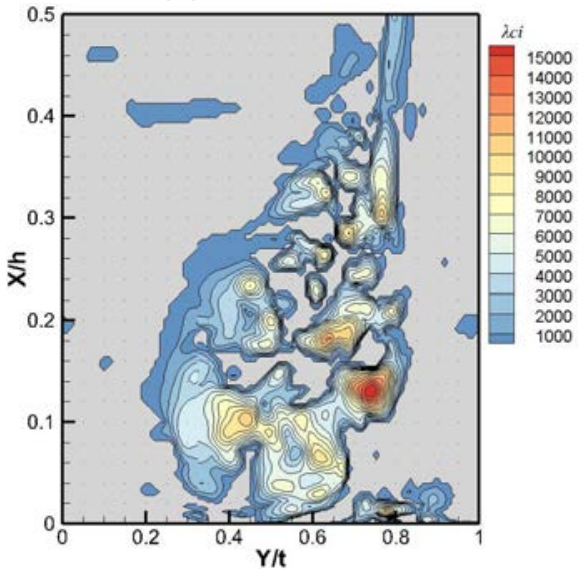

(e) $\lambda_{c i}$ criterion (d) $L T_{c r i}$ ER indication

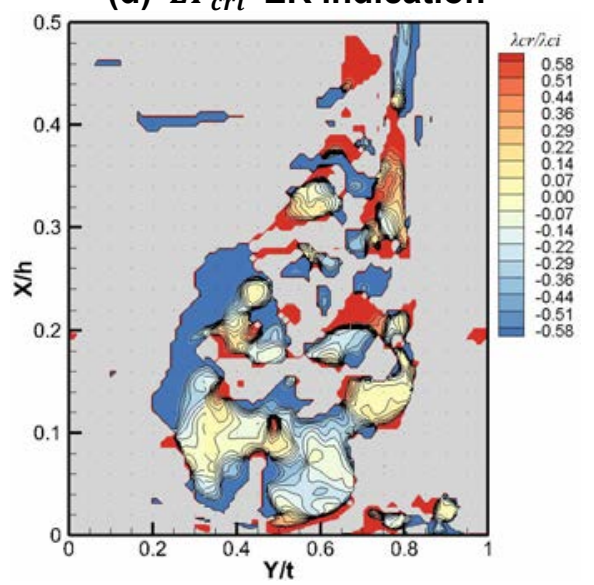

(f) $\lambda_{c r} / \lambda_{c i}$ criterion

Figure 6 Comparison of Different Vortex Identification in S3 Stream Surface

When comparing the difference between $Q$ criterion and $Q_{D}$ criterion, in association with $L T_{c r i}$ based ER (LTER) indication, it shows that major difference exists in the region whose projection in the ellipse is above the $\mathrm{P}=0$ line, namely, the flow compressibility for these region is expansion. Both the high threshold of $Q$ criterion and $Q_{D}$ criterion captures fewer vortices in this case.

Although the region recognised by $Q_{M}>0$ is much smaller than other methods, it commendably detaches the core vortex region observed from strain-rate principal axes. It contains less contaminate from shear. Regions with high magnitude of $Q_{M}$ also share high magnitude of $Q$ values, while not all the regions with high magnitude $Q$ values owe high $Q_{M}$ values. Observations from strain-rate principal axes are more objective. However, it also faces the problem of threshold choice. $Q$ criterion, $Q_{D}$ criterion and $L T_{c r i}$ based ER indication identifies the vortex in the original coordinate frame, according to the $\operatorname{tr}\left(\boldsymbol{S}^{2}\right)$ and $-\operatorname{tr}\left(\boldsymbol{\Omega}^{2}\right)$ distribution shown in Figure 5, these methods fail to recognize the region where the strain rate magnitude and vorticity magnitude is comparative. When the frame is changed into strain-rate principal axes, the region where the strain rate magnitude and vorticity magnitude is comparative while the vorticity magnitude is a bit more dominate is well detached by $Q_{M}$ method. It filters the influence of high shear.

Figure 6 (e) shows that the peak intensity of $\lambda_{c i}$ criterion relates well with $Q$ criterion and $Q_{D}$ criterion. However, $\lambda_{c i}$ criterion with positive threshold identifies larger number of vortices. Associated with LTER indication, it could be proved that the vortices missed by $Q$ criterion shares weak swirling tendency that can be excluded in the vortex core region. Obviously, the set for $\lambda_{c i}>0$ and $\Delta>0$ is exactly the same, thus, all the flow points that achieve $\lambda_{c i}>0$ could find its place in the projection plane (also termed as positive-Delta space) for LTER indication. The elliptical region that confined by $L T_{c r i}>0$ is a subset of the projection plane. Thus, $L T_{c r i}>0$ is a subset of $\lambda_{c i}>0$, and the same goes for $Q>0$ who also extract the vorticity dominated region from its constructed intention. $\lambda_{c i}>0$ and $\Delta>0$ are the least restrictive identifications.

The upper and lower threshold bound for $\lambda_{c r} / \lambda_{c i}$ enhanced swirling-strength criterion are suggested as $\pm 1 / \sqrt{3}$ (Chakraborty et al., 2005), and the suggested threshold bound is applied in Figure 6 (f). The region it confined contains more than the $Q$ criterion, but less than the $\lambda_{c i}$ criterion. Compared with $Q$ criterion, the disparities mostly exist in the region that extracted by the threshold near upper and lower bound. 
Figure 7 plots the distribution of $\operatorname{tr}\left(\boldsymbol{S}^{2}\right)$ and $-\operatorname{tr}\left(\boldsymbol{\Omega}^{2}\right)$ in the S1 stream surface at $10 \%$ span-height. The same legend is used as Figure 5. The high vorticity region (shown in green) also shares high magnitude of strain rate intensity, both of them supply each other. The vorticity dominated region appears in the separated region with a branch of structures that similar to the Karman vortex street.

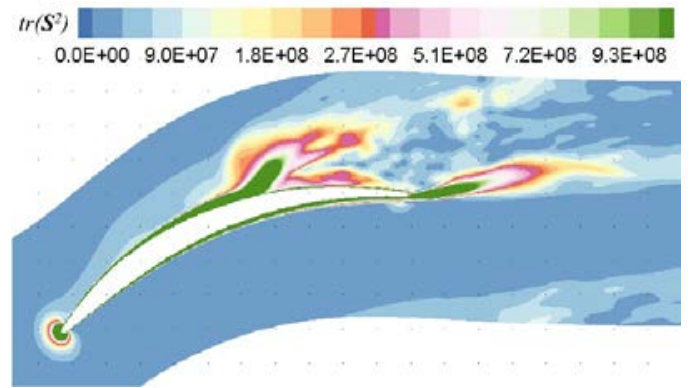

(a) $\operatorname{tr}\left(S^{2}\right)$

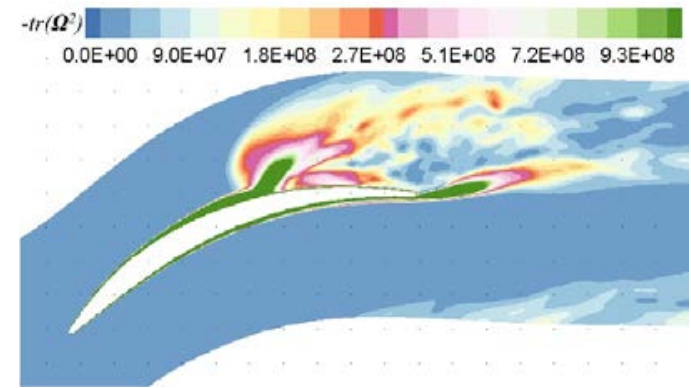

(b) $-\operatorname{tr}\left(\Omega^{2}\right)$

Figure 7 Distribution of $\operatorname{tr}\left(S^{2}\right)$ and $-\operatorname{tr}\left(\Omega^{2}\right)$ in $S 1$ Stream Surface

Similar to Figure 6, Figure 8 provides vortex identification results by $Q$ criterion, $Q_{D}$ criterion, $Q_{M}$ criterion, $L T_{c r i}$ based ER indication, $\lambda_{c i}$ swirling-strength criterion, and $\lambda_{c r} / \lambda_{c i}$ enhanced swirling-strength criterion in $\mathrm{S} 1$ stream surface at $10 \%$ height of span. The same legend and blanking manner is utilized as Figure 6.

Seen from S1 stream surface view, vortices in the practical flow field tend to overlap with each other, and have irregular edges. Analogously, comparison of Figure 8 (a) and Figure 8 (b) shows that none significant discrepancies are found by the identification results by $Q$ criterion and $Q_{D}$ criterion. High magnitude region of $Q_{M}$ criterion accords confines less vortex regions, excluding weak vortices that contaminate by large strain. LTER indication provides abundant swirling pattern information in the cross section of vortices in S1 stream surface. Vortices tear from the blade suction surface near the blade leading-edge appear in expansion state, while vortices within the blade passage mostly appear in compression state. Vortex structures identified by high values of $\lambda_{c i}$ criterion reveals a good correspondence to the high intensity of $-\operatorname{tr}\left(\Omega^{2}\right)$ distribution. As shown in Figure $8(\mathrm{f})$, contaminate is easy to induce by high magnitude of $\lambda_{c r} / \lambda_{c i}$ criterion in comparison to other identifications. The small quantity that added in the denominator to make the expression sense also add in the noise.

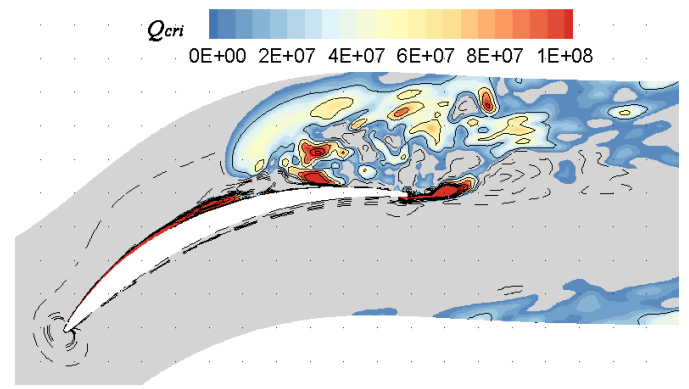

(a) $Q$ criterion

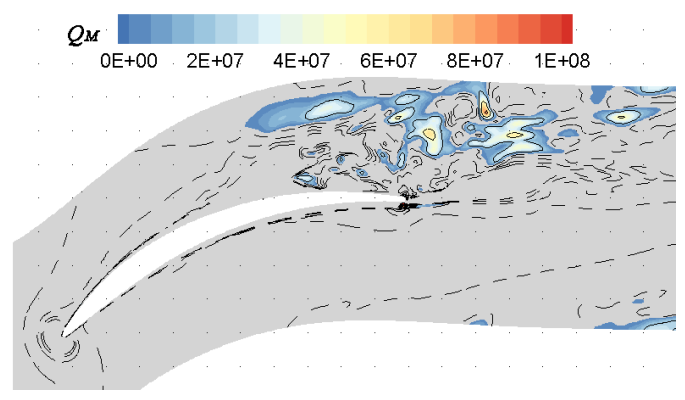

(c) $Q_{M}$ criterion

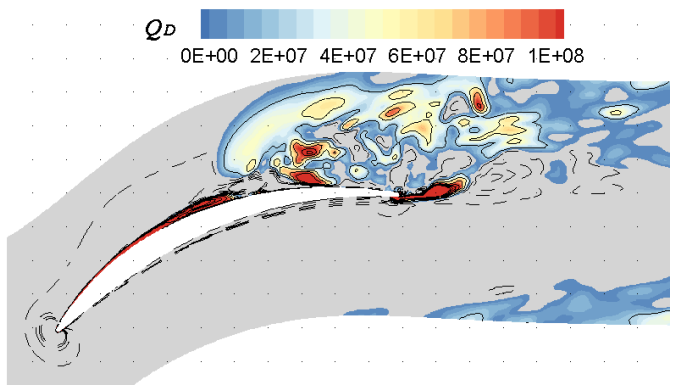

(b) $Q_{D}$ criterion

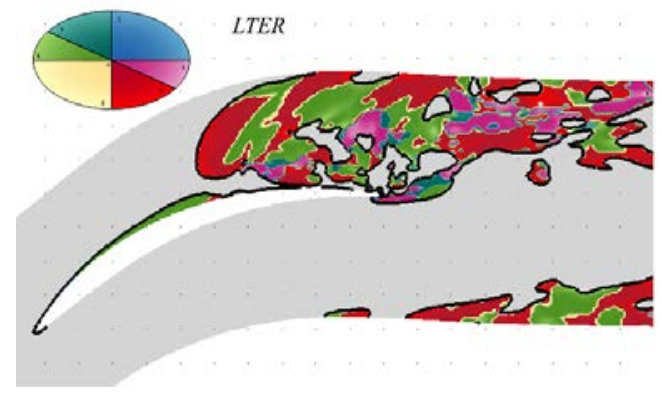

(d) $L T_{c r i}$ ER indication 


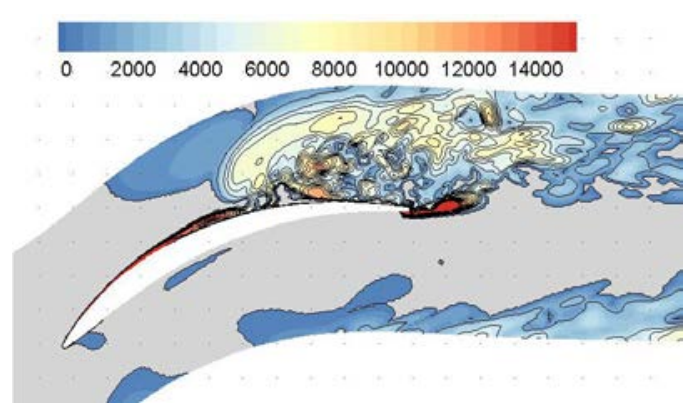

(e) $\lambda_{c i}$ criterion

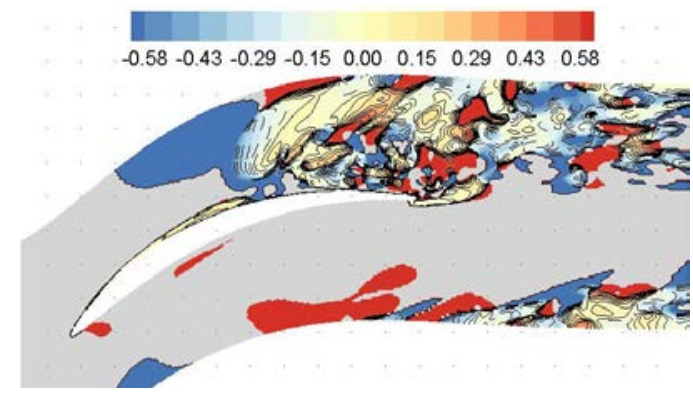

(f) $\lambda_{c r} / \lambda_{c i}$ criterion

Figure 8 Comparison of Different Vortex Identification in S1 Stream Surface

Non-zero thresholds are usually employed for most of vortex identification methods in practical use to extract the core region that regarded as stronger vortices. However, through the comparisons above, arbitrary choice of large threshold without precise physical meanings does not filter the weak vortices, for example, high magnitude of $Q$ criterion means the absolute difference between the intensity of vorticity and the intensity of strain rate is high, but there is none evidence to show it as a feature for strong vortex. High background shear effect and viscous diffusion make obstacles for identifying the vortex. Figure 6 (a), (b) and Figure 8 (a), (b) demonstrate that high $Q_{M}$ criterion threshold filters shear contaminated vortices that contained by $Q$ criterion and $\lambda_{c i}$ criterion. The LTER indication aims at distinguishing different swirling patterns, therefore shunning the problem of threshold choice. Opposite to most of identifications, vortices likely to exist in lower threshold of $\left|\lambda_{c r} / \lambda_{c i}\right|$ region.

\section{CONCLUSIONS}

To evaluate the capacity of different vortex identifications in extracting vortices in a highly loaded compressor cascade passage, the present study derives and presents the analytic solutions for different vortex identifications, both for the methods based on Helmholtz decomposition and its variation, and the methods based on eigen decomposition. The $L T_{c r i}$ based elliptical region indication that proposed by us recently is reviewed, and the inter-relationship between the Helmholtz decomposition series and the eigen decomposition series is built theoretically through $L T_{c r i}$ associates with the projection plane constructed for it. Then, the similarity and difference of identifications made by $Q$ criterion, $Q_{D}$ criterion, $Q_{M}$ criterion, $L T_{c r i}$ based elliptical region (ER) indication, $\lambda_{c i}$ swirling-strength criterion, and $\lambda_{c r} / \lambda_{c i}$ enhanced swirling-strength criterion are analysed in a DDES sampled flow field of a highly loaded compressor cascade. Both the vortices in S3 stream surface and S1 stream surface are extracted and compared.

Results show that vortices in the practical flow field tend to overlap with each other and have irregular edges. $Q_{D}$ criterion provides similar results to $Q$ criterion when the effect of compressibility is less. Both high threshold for $Q$ criterion and $Q_{D}$ criterion captures more vortices in this case. The region recognised by $Q_{M}>0$ is much smaller than other methods, and it is more objective since it observes from strain-rate principal axes, hence, the $Q_{M}$ criterion is good at filtering the influence of high background shear effect and viscous diffusion. However, $Q$ criterion and two of its variation forms all face the problem of threshold choice. Of all the methods compared, $\lambda_{c i}$ criterion with positive threshold identifies larger number of vortices, and structures identified by high values of $\lambda_{c i}$ criterion reveals a good correspondence to the high intensity of $-\operatorname{tr}\left(\Omega^{2}\right)$ distribution. $\lambda_{c i}>0$ and $\Delta>0$ are the least restrictive identifications, $L T_{c r i}>0$ is a subset of $\lambda_{c i}>0$, and the same goes for $Q>0$. LTER indication contributes to providing abundant swirling pattern information in the cross section of identified vortex structures. Contaminate is easy to induce by high magnitude of $\lambda_{c r} / \lambda_{c i}$ criterion in comparison to other identifications, and vortices trend to exist in lower threshold of $\left|\lambda_{c r} / \lambda_{c i}\right|$ region. Arbitrary non-zero thresholds should be avoided in seeking for the stronger vortices without precise physical meanings.

\section{NOMENCLATURE}

$\begin{array}{ll}c & \text { chord } \\ L T_{c r i} & \text { local trace criterion } \\ P, Q, R & \text { Symbols for the first, second, and third invariant of the velocity gradient } \nabla \boldsymbol{u} \\ Q_{c r i} & Q \text { criterion } \\ \boldsymbol{S} & \text { strain-rate tensor } \\ \nabla \boldsymbol{u} & \text { velocity gradient } \\ Y_{1}, Y_{2} & \text { auxiliary variables }\end{array}$


Greek symbols

$\Delta$

$\xi$

$\eta$

$\lambda_{c i}$

$\lambda_{c r}$

$\lambda_{r}$

$\boldsymbol{\Omega}$

Acronyms

CFD

ER

LT

LTER
$\Delta$ criterion/ discriminant of the velocity gradient tensor

ratio of $\lambda_{r} /\left|\lambda_{c i}\right|$

ratio of $\lambda_{c r} /\left|\lambda_{c i}\right|$

imaginary part of the complex eigenvalue of the velocity gradient tensor

real part of the complex eigenvalue of the velocity gradient tensor

real eigenvalue of the velocity gradient tensor when $\Delta>0$

vorticity tensor

computational fluid dynamics

elliptical region

local trace

$L T_{c r i}$ based elliptical region

\section{ACKNOWLEDGMENTS}

We express our appreciation to the late Prof. Lipeng Lu, whose contribution to this work was of great significance. This work is support by the National Natural Science Foundation of China (No. 51676007, No. 51976006, No. 51790513), the Aeronautical Science Foundation of China (2018ZB51013).

\section{References}

Berdahl C.H., Thompson D.S. (1993). Eduction of Swirling Structure Using the Velocity Gradient Tensor. AIAA Journal, 31(1), pp. 97-103

Chakraborty P., Balachandar S., Adrian R.J. (2005) On the Relationship Between Local Vortex Identification Schemes. Journal of Fluid Mechanics, 535, pp. 189-214.

Chong M.S., Perry A.E., Cantwell B.J. (1990). A General Classification of Three-Dimensional Flow Fields. Physicals of Fluids A: Fluid Dynamics, 2(5), pp. 765-777.

Epps B.P. (2017). Review of Vortex Identification Methods. The 55th AIAA Aerospace Sciences Meeting, AIAA $2017-$ 0989

Fan S.J. (1989). A new extracting formula and a new distinguishing means on the one variable cubic equation, Journal of Hainan Normal University (Natural Sci.), 2, pp. 91-98 (in Chinese).

Gbadebo S.A., Cumpsty N.A., Hynes T.P. (2005). Three-Dimensional Separation in Axial Compressors. ASME Journal of Turbomachinery, 127(2), pp. 331-339.

Gregory S.D., Graves C.P. (1983). Secondary Flows and Losses in a Turbine Cascade. England: Durham University. Herzig H.Z., Hansen A.G., Costello G.R. (1954). A Visualization Study of Secondary Flows in Cascades. United States: NACA Annual Report, 40, pp. 147-197.

Hunt J.C.R., Wray A.A., Moin P. (1988). Eddies, Streams and Convergence Zones in Turbulent Flows. Center for Turbulence Research Proceedings of the Summer Program, pp. 193-208

Kang S. (1993). Investigation of the Three Dimensional Flow within a Compressor Cascade with and without Tip Clearance. PhD. Brussel: Vrije University.

Klein A. (1966). Investigation of the Entry Boundary Layer on the Secondary Flows in the Blading of Axial Turbine. BHRAT1004.

Kolář V. (2009). Compressible Effect in Vortex Identification. AIAA Journal, 47(2), pp. 473-475.

Kolář V., Śístek J. (2015). Corotational and Compressibility Aspects Leading to a Modification of the VortexIdentification Q-criterion. AIAA Journal, 53(8), pp. 2406-2410

Liu Y.W., Tang Y.M. (2019a). An Elliptical Region Method for Identifying a Vortex with Indications of Its Compressibility and Swirling Pattern. Aerospace Science and Technology, 95, p. 105448

Liu Y.W., Zhong L.Y., Lu L.P. (2019b). Comparison of DDES and URANS for Unsteady Tip Leakage Flow in an Axial Compressor Rotor. ASME Journal of Fluids Engineering, 141(12), p. 121405.

Liu Y.W., Yan H., Lu L.P., Li Q.S. (2017). Investigation of Vortical Structures and Turbulence Characteristics in Corner Separation in a Linear Compressor Cascade Using DDES. ASME Journal of Fluids Engineering, 139(2), p. 021007

Tang Y.M., Liu Y.W., Lu L.P., Lu H.W., Wang M. (2017) Experimental Investigation of Flow Control Using Blade End Slots in a Highly Loaded Compressor Cascade. The 17th International Symposium on Transport Phenomena and Dynamics of Rotating Machinery, December 16-21 2017, Maui, Hawaii, USA.

Tang Y.M., Liu Y.W., Lu L.P. (2018). Solidity Effect on Corner Separation and Its Control in a High-Speed Low Aspect Ratio Compressor Cascade. International Journal of Mechanical Sciences, 142, pp. 304-321.

Tang Y.M., Liu Y.W., Lu L.P., Lu H.W., Wang M. (2020). Passive Separation Control with Blade-End Slots in a Highly Loaded Compressor Cascade, AIAA Journal, 58(1), pp. 85-97.

Tucker P.G. (2013). Unsteady Computational Fluid Dynamics in Aerodynamics. Springer Science \& Business Media. 